\title{
Réorganisation de la pratique pharmaceutique en établissement de santé : le cas de l'Hôpital Saint-Michel de Jacmel en Haïti
}

\author{
Jean-François Bussières, Viriginie Bussières, Jean Lafontant Maurice et Martine Legault
}

\section{INTRODUCTION}

$\mathrm{H}$

aïti est un pays francophone des Caraïbes avec qui le Québec entretient des liens privilégiés, compte tenu des besoins criants de la société haïtienne découlant notamment du séisme de 2010, du partage d'une langue commune, le français, et d'une diaspora établie au Québec.

Le Programme de Santé Intégré en Haïti (PSI) est une initiative de la Croix-Rouge Canadienne et de la Croix-Rouge Haïtienne. Trois partenaires participent à la réalisation du projet, soit le Centre hospitalier universitaire (CHU) Saint-Justine, la Direction de la santé publique de l'Agence de la santé et des services sociaux de Montréal et l'Unité de santé internationale de l'Université de Montréal. Le CHU Sainte-Justine est notamment responsable de l'implantation d'un programme d'amélioration de la qualité de la prestation des soins et services, dont fait partie la pharmacie. Le Programme cible la réorganisation et la reconstruction de l'Hôpital Saint-Michel de Jacmel, situé dans le département du Sud-Est haïtien ${ }^{1}$. Lancé en décembre 2011, ce projet s'échelonne sur une période de cinq ans.

L'objectif de cet article est de présenter une démarche de réorganisation des services et des soins pharmaceutiques en Haïti.

\section{MÉTHODE}

Il s'agit d'une étude descriptive observationnelle. Elle se déroule à l'Hôpital Saint-Michel de Jacmel. Ce département, d'une population d'environ 575000 habitants, compte 63 institutions sanitaires, un hôpital départemental (l'hôpital SaintMichel), 689 professionnels de la santé et quatre pharmaciens. L'Hôpital Saint-Michel, qui a été passablement affecté par le séisme de 2010 (c.-à-d. destruction de plusieurs bâtiments, dont trois unités de soins et le bloc opératoire), comporte un service des urgences, une clinique externe, une clinique de vaccination, un centre de déclaration volontaire pour le VIH/SIDA, deux salles d'opération, un service de médecine interne, un service de chirurgie, un service de pédiatrie, un service de maternité et obstétrique, un centre de transfusion et une pharmacie. Au moins deux des services sont situés dans des bâtiments temporaires mis en place par Médecins sans frontières après le séisme. Outre la destruction de bâtiments, le séisme a provoqué la désorganisation de l'institution, certains de ses employés ayant quitté la ville et l'établissement en raison des nombreuses difficultés auxquelles ils étaient confrontés ( $p$. ex. crise financière majeure, hygiène menacée à cause de la destruction des infrastructures, présence de vermine et difficulté de gérer les déchets). L'Hôpital accueille environ 3200 patients par année. On note environ 1700 accouchements et 240 interventions chirurgicales.

Le service de pharmacie compte un pharmacien, une coordonnatrice du circuit du médicament et du matériel de soins, six régisseuses (l'équivalent d'assistant technique) et une personne s'occupant de l'entretien. La pharmacie est ouverte 24 heures par jour et doit normalement fournir l'ensemble des médicaments requis durant les hospitalisations. Le circuit du médicament n'est pas placé sous la responsabilité de la pharmacie, qui ne fait que dispenser les médicaments à la demande. L’organisation des réserves d'étage est laissée au personnel soignant. Les produits médicamenteux sont stockés dans des armoires métalliques ou de bois. Il n'existe aucun système de classement uniformisé, et les réserves comportent de nombreux médicaments périmés, classés pêle-mêle avec le matériel de soins et les autres équipements dans des conditions hygiéniques insuffisantes. L'hygiène globale laisse aussi à désirer. La pharmacie comporte trois pièces, soit la pharmacie principale (qui est composée d'une pièce d'environ 60 mètres carrés, non climatisée comprenant un comptoir de service, un réfrigérateur, un bureau et des étagères), une pièce d'environ 20 mètres carrés pour l'entreposage sécurisé et une pièce d'environ 20 mètres carrés comprenant le bureau du pharmacien, le lit de garde et des toilettes. Le stock de médicaments est géré partiellement à l'aide du logiciel Channel fourni depuis 2009 par United Nations Populations Fund. La distribution des médicaments s'effectue à partir d'ordonnances individuelles nominatives. Les médicaments sont remis à la 
personne dans des sacs en plastique de type Ziploc et sont accompagnés d'une description minimale ainsi que d'une facturation manuscrite. La pharmacie reçoit de 30 à 80 ordonnances par jour. Compte tenu de la crise actuelle, une proportion significative des ordonnances ne peut être traitée, et les patients (ou plutôt les personnes qui les accompagnent durant leur séjour hospitalier) sont redirigés vers les pharmacies de ville. Certains patients se dirigent parfois vers des pharmacies ambulantes, où il n'existe aucun encadrement (c.-à-d. médicaments exposés à la lumière et à une chaleur intense, provenance incertaine, aucun professionnel formé et vente à des prix variables sans aucune traçabilité). La pharmacie ne tient aucun dossier patient.

L'étude repose sur une première mission de reconnaissance réalisée en octobre 2013 et sur une seconde mission de réorganisation réalisée en février 2014. La démarche est décrite de façon qualitative afin de soutenir d'autres initiatives similaires. Pour chaque mission, le pharmacien du CHU Sainte-Justine était accompagné d'un médecin haïtien coordonnateur embauché par le CHU Sainte-Justine afin de faciliter les contacts et les observations.

Lors de notre première mission de reconnaissance de sept jours en octobre 2013, nous avons effectué une revue documentaire sur l'état des lieux en matière de pratique pharmaceutique en Haïti ${ }^{2,3}$. Elle a permis de situer le pays, l'organisation de son système de santé, le marché du médicament et les initiatives humanitaires de type pharmaceutique.

Afin de bien saisir l'état de la pratique pharmaceutique, nous avons visité l'autorité départementale ainsi que le pharmacien désigné, le Centre départemental d'approvisionnement en intrants, un centre de santé avec des lits (Marigot), un dispensaire (Marbial), deux autres hôpitaux publics (cubain, protestant), un hôpital privé (clinique du Dr Martinez) et un tradipraticien (c.-à-d. une personne formée en médecine traditionnelle par un pair, le plus souvent par tradition orale). Le pharmacien du CHU Sainte-Justine a rencontré en outre le directeur général, le chef pharmacien et son équipe, les infirmières responsables de services, les médecins, le logisticien, le chef comptable et l'administrateur.

\section{RÉSULTATS}

Au terme de cette première mission, un rapport a mis en évidence de nombreux problèmes : un cadre juridique limité encadrant l'exercice de la pharmacie, un cadre financier incertain, une tradition orale très ancrée (aucun document imprimé), une pénurie de données financières, de financement, de médicaments et de matériel de soins, des problèmes d'organisation et de salubrité, tant à la pharmacie que dans les services.

Des travaux préparatoires ont été amorcés en vue d'une seconde mission, notamment le choix et l'acquisition de matériel visant la réorganisation informatique, l'aménagement des réserves d'étage, la réflexion sur l'organisation du " nouveau " service de pharmacie, l'exploration des possibilités d'utilisation du codebarres et l'acquisition de médicaments à titre de dons.
Une seconde mission de réorganisation de 18 jours a eu lieu. Un pharmacien du CHU Sainte-Justine aidé d'une stagiaire en coopération internationale ont d'abord effectué une revue documentaire complémentaire afin de comprendre le rôle du United Nations Population Fund et de son logiciel Channel utilisé en gestion logistique pour les médicaments et le matériel de soins. Ils ont consulté en outre les travaux d'évaluation du Programme de pharmacie à la Faculté de médecine et de pharmacie de l'Université d'État d'Haïti, l'état des travaux et les réalisations dans le domaine de la santé en Haïti en 2013, les ressources documentaires scientifiques pertinentes sur l'aide humanitaire et les sources de dons de médicaments pour les professionnels de la santé venant du Canada.

Les deux missions ont établi un relevé quotidien des observations et des personnes de contact, accompagné de photos et de vidéos.

Le ministère de la Santé publique et de la Population en Haïti utilise une fiche d'inspection institutionnelle. La cotation de cette fiche indiquait un faible taux de conformité à la majorité des critères. Avec la mission de réorganisation tenue en février 2014, au moins 25 des 110 critères ont fait l'objet d'une amélioration significative.

Le tableau 1 présente le profil des recommandations faites lors de la mission de reconnaissance en octobre 2013, l'état des réalisations accomplies lors de la deuxième mission de réorganisation en février 2014 et le plan des actions subséquentes.

\section{DISCUSSION}

Il existe relativement peu de données sur la contribution des pharmaciens humanitaires ${ }^{4-10}$. Au moins trois organismes francophiles soutiennent des initiatives pharmaceutiques humanitaires, soit Pharmaciens humanitaires fondé en $2001^{11,12}$, Pharmaciens sans frontières Canada fondé en $1995^{13}$ et ReMeD (Réseau médicaments et développement) ${ }^{14}$. Notre étude met en évidence une démarche intuitive, fondée sur l'observation, la détection des problèmes et leur résolution.

Il existe relativement peu de balises internationales sur l'organisation des services et des soins pharmaceutiques en établissement de santé. L'énoncé de Basel, publié sous l'égide de la Fédération internationale pharmaceutique (FIP) a permis la publication en 2009 d'un consensus sur le futur de la pharmacie hospitalière. Cette entente comporte sept axes et 75 articles qui peuvent être utiles lors d'audits externes de pharmacie hospitalière ${ }^{15}$. Poh et coll. l'ont utilisée lors d'une réorganisation menée dans un hôpital en Ouganda ${ }^{10}$. Plus récemment en 2011, la FIP a publié conjointement avec l'Organisation mondiale de la santé (OMS) une version conjointe de ses bonnes pratiques pharmaceutiques ${ }^{16}$. Elle a aussi publié le guide "Élargir la pratique pharmaceutique : recentrer les soins sur les patients $»^{17}$. Ce cadre de référence met en valeur le rôle clinique du pharmacien dans la perspective de l'exercice d'une pharmacie orientée vers les résultats cliniques. Il faut aussi noter les principes directeurs de l'OMS en matière de dons de médicaments ${ }^{18,19}$ et le guide sur la contrefaçon $^{20}$. L'ensemble de notre démarche tient compte de ces orientations 
This single copy is for your personal, non-commercial use only.

For permission to reprint multiple copies or to order presentation-ready copies for distribution, contact CJHP at cjhpedit@cshp.ca

\section{Tableau 1. Recommandations, réalisations et actions découlant des missions en Haïti}

Recommandations (mission de reconnaissance en octobre 2013)

№ 1 - Établir le profil financier des dépenses totales de l'établissement de santé, de la part des revenus publics et privés (patients) et de la valeur des exonérations.

\section{Réalisations (mission de réorganisation en février 2014)}

Non atteint

Il est difficile d'établir un profil financier sans informatisation (en cours). Conformément à la demande, nous avons isolé les revenus et les dépenses de la pharmacie dans un centre d'activités distinct afin d'assurer le suivi financier. L'établissement ne peut acheter de médicaments sans générer des revenus équivalents issus de leur vente; l'établissement ne reçoit quasiment aucun financement public pour ses intrants.

№ 2 - Compte tenu du contexte financier et juridique, clarifier les règles de couverture des soins de santé prodigués par I'HSMJ (décembre 2013).

\section{Atteint en grande partie}

Rédaction de politiques et de procédures permettant de fournir sans frais la $1^{\text {re }}$ dose de médicaments et de matériel de soins à partir des réserves d'étage; toute dose subséquente est vendue par le pharmacien avec une marge bénéficiaire de départ de $20 \%$ (achat régional) ou de $30 \%$ (achat dans la capitale). Impression des grilles tarifaires manuscrites sur de multiples documents peu lisibles.

\begin{tabular}{|c|c|}
\hline $\begin{array}{l}\text { No } 3 \text { - Compte tenu de la refonte en } \\
\text { cours de l'organisation sanitaire à la } \\
\text { Direction départementale, développer } \\
\text { un arrimage formel entre les pharmaciens } \\
\text { du département (comité régional sur } \\
\text { les services et les soins pharmaceutiques). }\end{array}$ & $\begin{array}{l}\text { Non atteint } \\
\text { Le département du Sud-Est compte en } \\
\text { théorie quatre pharmaciens selon l'état des } \\
\text { ressources; dans les faits, il n'en reste que } \\
\text { deux (le pharmacien départemental en } \\
\text { charge de } 63 \text { installations et le pharmacien } \\
\text { de l'HSMJ); il était peu réaliste d'établir un } \\
\text { comité régional en raison de la pénurie de } \\
\text { ressources à court terme. }\end{array}$ \\
\hline
\end{tabular}

№ 3 - Compte tenu de la refonte en cours de l'organisation sanitaire à la du département (comité régional sur ressources à court terme.
Achever la révision de la grille tarifaire hospitalière (toutes les activités chirurgicales et cliniques facturables) et pharmaceutique (prix de vente de chaque médicament) à partir de la mise à jour informatique de la gestion des stocks de la pharmacie déjà amorcée.
Actions (mission de
réorganisation de février 2014) Terminer l'informatisation de la comptabilité et établir un profil financier des revenus et des dépenses, y compris la valeur des dépenses issues des patients exonérés (à la discrétion de la direction générale, compte tenu de l'incapacité de payer).
À réaliser avec le pharmacien remplaçant lorsqu'il sera identifié compte tenu du départ évoqué du pharmacien de l'HSMJ. internationales, mais s’inspirent aussi de la pratique canadienne.

La plupart des orientations internationales ciblent les activités de sélection, d'approvisionnement et de distribution des médicaments, ainsi que de la gestion de leurs déchets. En tenant compte de la perspective canadienne, les changements proposés à l'Hôpital Saint-Michel de Jacmel visent à décentraliser le pharmacien pour le placer dans les services, pour favoriser tant la supervision du circuit du médicament qu'une éventuelle contribution aux activités cliniques. À titre d'exemple, une proportion significative de patients ne reçoit pas l'ensemble des doses prescrites, compte tenu de la grave pénurie de médicaments et des ressources financières limitées de l'institution et des patients. En effet, selon un audit de dossiers, les patients n'ont pas reçu $50 \%$ des doses d'antibiotiques injectables, des antihypertenseurs ou des antidiabétiques nécessaires. Par ailleurs, la répartition des médicaments ayant fait l'objet de dons et apportés directement par différents professionnels de la santé est anarchique; ils sont entreposés de manière désordonnée dans les réserves d'étage. Cette situation empêche l'utilisation optimale des médicaments. Il a suffi de quelques visites du pharmacien dans les services pour mettre en évidence la nécessité de regrouper tous les médicaments à la pharmacie, de rédiger et de diffuser une liste des dons reçus et de participer aux décisions entourant leur meilleur usage. Il faut toutefois convenir que la formation actuelle locale ne prépare pas les pharmaciens haïtiens à ce rôle clinique décentralisé. Alors que la Faculté de médecine et de pharmacie de l'Université d'État d'Haïti réfléchit à une refonte de son cursus, nous pensons qu'il est possible et souhaitable d'utiliser le terreau de ce projet de réorganisation à Jacmel, pour explorer la décentralisation du pharmacien dans les services.

Notre étude met en évidence la nécessité de réorganiser l'ensemble du circuit du médicament. Compte tenu des ressources en place et de la reconstruction d'un nouvel hôpital, nous avons choisi de ne pas attendre la disponibilité du nouveau bâtiment pour réorganiser l'activité pharmaceutique et clinique. Ainsi, on observe encore des écarts de pratique tant à la pharmacie que dans les services, plusieurs de ceux-ci étant susceptibles d'être corrigés avec la construction de deux nouveaux bâtiments, tels que hygiène, l'électricité en continu, la chaîne thermique, la sécurisation des stocks et l'informatisation des dossiers patients. Les prochaines missions permettront sans doute de déterminer en outre les actions complémentaires à entreprendre, deux missions par année étant prévues pour les deux ou trois prochaines années. Compte tenu de l'arrimage établi avec lesautorités nationales, nous pensons que les succès vécus à l'échelle de cette institution pourraient avoir des retombées à plus large échelle dans le pays. 
This single copy is for your personal, non-commercial use only.

For permission to reprint multiple copies or to order presentation-ready copies for distribution, contact CJHP at cjhpedit@cshp.ca

Tableau 1. Recommandations, réalisations et actions découlant des missions en Haïti (suite)

Recommandations (mission de reconnaissance en octobre 2013)

$\mathrm{N}^{\circ} 4$ - Mettre en place un nouveau département de pharmacie arrimé à la nouvelle structure de l'HSMJ.

Un chef de département à temps complet, un pharmacien en service social à temps complet et cinq régisseurs à temps complet. Planifier l'ajout d'un régisseur à la pharmacie pour une gestion décentralisée médicament) : des stocks.

Il semble très souhaitable d'afficher le poste de chef du nouveau département de pharmacie et de permettre à tous les candidats intéressés de postuler :

- La rédaction de politiques et de procédures afin de mieux structurer les processus ( $p$. ex. achats, accès, entretien, liste de médicaments locale, réserves d'étage, facturation aux patients, déchets pharmaceutiques); les faibles ressources matérielles limitent la capacité d'imprimer des documents « durables »;

- La rédaction de descriptions de tâches claires qui tiennent compte du référentiel national et des besoins de I'HSMJ, afin notamment de clarifier les rôles, d'accroitre la délégation aux régisseuses (y compris la double vérification de certains processus) et de décentraliser le pharmacien dans les unités de soins;

- La réorganisation de la gestion des stocks, comprenant l'établissement de listes des médicaments autorisés dans les réserves d'étage et la du personnel de pharmacie pour le stocks provenant de dons humanitaires. le public;

\section{Réalisations (mission de réorganisation en février 2014)}

\section{Atteint en grande partie}

Rédaction et impression de plusieurs

politiques et procédures intégrées à un

cartable départemental (organisation du service de gestion des médicaments et

fournitures de soins, facturation, gestion des stocks, gestion du circuit du

- Rédaction de descriptions de tâches et création du titre d'emploi de coordonnateur du circuit du médicament et des fournitures de soins;

- Tenue d'une première réunion de service;

- Mise à niveau du poste informatique du pharmacien avec réseau wi-fi et don d'un portable et d'une imprimante laser pour l'informatisation du poste de coordonnateur du circuit du médicament;

- Prise du stock global par la pharmacie;

- Nettoyage et peinture de la pharmacie, y compris affichage avec babillard, réparation du mobilier;

- Retrait pour incinération des produits au court délai de péremption; élimination d'un accès possible à ces produits par

- Grand ménage et réorganisation d'une réserve centralisée (deux étages) et des réserves d'étages comprenant les dons et les achats locaux de contenants de deux des six services de soins avec standardisation des produits disponibles et mise en place d'un processus plus structuré impliquant la décentralisation du personnel de pharmacie pour le réapprovisionnement;

- Révision des pratiques entourant l'hygiène et le classement;

- Tenue d'une première réunion avec les infirmières responsables de services;

- Ajout de matériel scientifique de MSF.

No 5 - Trouver le financement suffisant pour I'hébergement du pharmacien en service social.

\section{Non atteint}

La Direction générale de HSMJ n'a fait

Sud-Est (et MSPP) pour trouver un

pharmacien en service social; ceci a été

vérifié personnellement avec la directrice de la DPM/MT.

\section{Actions (mission de réorganisation de février 2014)}

Cette recommandation demeure pertinente; il faut encore :

- Procéder à l'ajout d'une régisseuse:

- Remplacer le réfrigérateur de la pharmacie et revoir le processus de chaîne thermique pour approvisionner les services et conserver les médicaments;

- Compléter le réaménagement des buffets des urgences et salle d'opération;

- À partir du restockage et de la mise à jour de Channel, établir les paramètres de minimum-maximum pour limiter les pénuries;

- Établir un mécanisme de diffusion des stocks disponibles pour le personnel soignant et pour le prescripteur pour la portion tarifée;

- À moyen terme, envisager la saisie des ordonnances dans un logiciel libre de droits (open source).

№ 6 - S'assurer que les aménagements Non atteint prévus dans le nouvel hôpital permettent Plans non disponibles sur place de faire fonctionner le nouveau département de pharmacie.

Cette recommandation demeure pertinente, et il faut procéder formellement à cette demande au département du Sud-Est à court terme et trouver le financement destiné à l'hébergement.

Cette recommandation demeure pertinente.

Il existe peu de ressources visant à préparer les pharmaciens à l'action humanitaire. Par exemple, la Faculté d'éducation permanente de l'Université de Montréal offre un certificat en coopération internationale ${ }^{21}$. Ce dernier a été développé en collaboration avec l'Association québécoise des organismes de coopération internationale, le Carrefour canadien international, le Centre d'étude et de coopération internationale, le Cuso
International, Équiterre, Jeunesse Canada Monde, OxfamQuébec, le Service d'assistance canadienne aux organismes et Développement et Paix. Des formations similaires existent dans quelques universités canadiennes.

Lintervention dans un pays en difficulté, en crise ou en réorganisation n'est pas simple. S'il faut s'imprégner de la culture et de ses structures, il faut aussi savoir observer, écouter, apprendre 
This single copy is for your personal, non-commercial use only.

For permission to reprint multiple copies or to order presentation-ready copies for distribution, contact CJHP at cjhpedit@cshp.ca

Tableau 1. Recommandations, réalisations et actions découlant des missions en Haïti (suite et fin)

Recommandations (mission de reconnaissance en octobre 2013)

№ 7 - Créer un comité du médicament et du matériel de soins (équivalent du comité de pharmacologie au Canada).

\section{Réalisations (mission de réorganisation en février 2014)}

Atteint en grande partie

- Rédaction de politiques et de procédures pour le comité;

- Identification des membres et formation du comité (trois médecins, trois infirmières, un pharmacien et la coordonnatrice du circuit du médicament);

- Tenue d'une première réunion du comité des médicaments et du matériel de soins avec échanges de courriels et comptes rendus; le comité se réunira une fois par mois sous la présidence du pharmacien;

- Adoption de politiques et procédures concernant les réserves d'étage.
№ 8 - Réorganiser la pharmacie et restocker de façon adéquate les produits et le matériel afin de soutenir la réorganisation du département de pharmacie.

No 9 - Développer un programme de formation pharmaceutique.

\section{Atteint en partie}

Remise de deux Physicians Travel Pack.

\section{Actions (mission de} réorganisation de février 2014)

Cette recommandation demeure pertinente

et comporte les points suivants:

- Mener une revue d'utilisation des médicaments prescrits afin d'avoir un profil de la prescription actuelle;

- Établir une liste locale des médicaments et du matériel de soins, en collaboration avec les chefs médicaux et s'assurer que ces produits sont réellement prescrits;

- Établir des règles d'utilisation pour le bon usage des médicaments, y compris l'indication, la posologie, les précautions et les contre-indications;

- Établir des règles en cas de pénurie (p. ex. substitution thérapeutique, utilisation de produits périmés en situation d'urgence;

- Établir des protocoles de traitement pour les situations critiques et fréquentes; vérifier l'applicabilité de protocoles existants (p. ex. OMS, MSF).

Cette recommandation demeure pertinente

- Apport de stocks complémentaires dans une prochaine mission;

- Le pharmacien local doit proposer deux commandes d'environ 5,000\$ pour le restockage complémentaire.

Cette recommandation demeure pertinente; le programme de formation envisagé comporte les éléments suivants:

- Formation sur l'accueil destinée aux étudiants en soins infirmiers pour la « bonne utilisation du circuit du médicament »;

- Formation de base du personnel de la pharmacie sur la « bonne gestion de la pharmacie »;

- Formation de base du personnel médical sur la bonne «prescription » de médicaments;

- Développer un stage humanitaire court de 4 semaines pour les étudiants et résidents en pharmacie à I'Université de Montréal.

No 10 - Arrimer le projet de refonte des services et soins pharmaceutiques de I'HSMJ avec le MSPP et la DPM/MT.

\section{Atteint en partie}

- Excellent arrimage du médicament et de la médecine traditionnelle avec la Direction de la pharmacie.
Cette recommandation demeure pertinente

- Participation au colloque de mars à Port-au-Prince afin de faire connaître la vision de la pharmacie hospitalière;

- Partage des travaux avec les autorités ministérielles afin d'accroître la pérennité de ces actions.

DPM/MT = Direction de la pharmacie, du médicament et de la médecine traditionnelle, HSMJ = Hôpital Saint-Michel de Jacmel, MSF = Médecins sans frontières, MSPP = ministère de la Santé publique et de la Population, OMS = Organisation mondiale de la Santé.

et comprendre. Plus encore, il faut savoir agir et mobiliser les personnes locales, dans le respect et le partage des mêmes objectifs. La participation à l'action humanitaire constitue une occasion formidable de remise en question personnelle et professionnelle, de validation de paradigmes (p. ex. gestion du changement, communication efficiente et cohérente, hygiène et organisation), de dépassement et de don.

Cette étude descriptive comporte des limites. Elle repose sur deux courtes missions de reconnaissance et d'action dans un hôpital haïtien. Le contexte politique, économique, social et 
sanitaire de chaque pays peut affecter significativement les objectifs envisagés et les actions entreprises. La réorganisation des services et des soins pharmaceutiques de l'hôpital ciblé nécessitera quelques années, et il faudra attendre pour mesurer pleinement les retombées des changements proposés. L'étude présente une perspective à court terme de l'action amorcée. Nous pensons toutefois qu'il est utile de rapporter ces initiatives, compte tenu du nombre limité de publications et des besoins immenses à l'échelle des pays en développement.

\section{CONCLUSION}

Il existe peu de données sur l'activité des pharmaciens en humanitaire. Cette étude descriptive met en évidence la démarche de réorganisation de la pratique pharmaceutique en établissement de santé à l'Hôpital Saint-Michel de Jacmel en Haïti. Dans le cadre d'une mission de reconnaissance de sept jours et d'une mission de réorganisation de 18 jours, nous avons décrit les activités réalisées et à venir. La contribution d'un pharmacien du CHU Sainte-Justine est prometteuse de changements pour rendre la pratique pharmaceutique en établissement de santé plus opérationnelle. Par ailleurs, la réussite de cette entreprise est directement liée à l'engagement des équipes sur place.

\section{Références}

1. Programme intégré pour la santé. Ottawa $(\mathrm{ON})$ : Croix-rouge canadienne; 2013. Publié au : www.redcross.ca/haiti2010/red-cross-response/health-program-f.asp. Consulté le 3 mars 2014.

2. Bussières JF. État des lieux en pratique pharmaceutique à Haiti - mise en contexte d'un pharmacien. Ann Unité Rech Prat Pharm. 2013 nov. 14:; 1-61. Publié au : http://indicible.ca/urpp/20131114_HAITI_Article_ Annales.pdf. Consulté le 3 mars 2014.

3. Bussières JF, Bussières V. État des lieux en pratique pharmaceutique à Haïti - mise en contexte d'un pharmacien - partie II. Ann Unité Rech Prat Pharm. 2014 mars 13:;1-19. Publié au : http://indicible.ca/urpp/20140313_ HAITI2_Annales.pdf. Consulté le 2 septembre 2014.

4. Ford ME. Faith, charity, and HOPE: pharmacists participate in humanitarian assistance to the former Soviet Union. Am J Hosp Pharm. 1992;49(6):1312, 1316-8

5. Roy H. Organisation d'une pharmacie dans un centre hospitalier à Oyem, Gabon. Pharmactuel. 2005;38(2):79-84.

6. Louz Y. Actions de pharmaciens sans frontière international, un exemple le cambodge. Med Trop. 2006;66(6):1-5.

7. Bonnabry P, Bengaly L, Pittet D. Perspectives de développement de la pharmacie hospitalière au Mali. Rev Med Suisse. 2006;2:S36-S38.

8. Bussières JF, St-Arnaud C, Schunck C, Lamarre D, Jouberton F. The role of the pharmacist in humanitarian aid in Bosnia-Herzegovina: the experience of Pharmaciens Sans Frontières. Ann Pharmacother. 2000;34(1):112-8.

9. Tardif L, Gallay E, Béland AJ. Pharmaciens sans frontière au Mali. Pharmactuel. 2007;40(4):224-7.

10. Poh J, Vaillancourt R, Lamarre D, Oyella J. Use of the 2008 Basel consensus statements to assess, realign, and monitor pharmacy practice at a tertiary care hospital in northern Uganda: illustrative case study. Can J Hosp Pharm. 2013;66(5):318-27.

11. Diplôme d'université. Pharmacie et aide humanitaire. Caen (France) : Université de Caen, Département de formation continue et professionnelle; 2012. Publié au : www.pharmahuma.org/wp-content/uploads/2012/04/ PAH-plaquette-DU.pdf. Consulté le 3 mars 2014.

12. PAH-Opérations. Caen (France) : Les Pharmaciens Humanitaires; 2013. Publié au : www.pharmahuma.org/qui-sommes-nous/nous-contacter. Consulté le 3 mars 2014.

13. Historique. Montréal (QC) : Pharmaciens sans frontières - Canada. Publié au : www.psfcanada.org/index.php?page=history. Consulté le 3 mars 2014.

14. Présentation. Paris (France) : ReMeD (Réseau médicaments et développement). Publié au : www.remed.org/html/le_reseau_remed.html. Consulté le 3 mars 2014 .
15. American Society of Health-System Pharmacists. The Basel Statements on the future of hospital pharmacy. Am J Health Syst Pharm. 2009;66(5 Suppl 3):S61-6.

16. Good pharmacy practice. La Haye (Pays-Bas) : Fédération internationale pharmaceutique. Publié au : www.fip.org/good_pharmacy_practice. Consulté le 3 mars 2014.

17. Weidenmayer K, Summers RS, Mackie CA, Gous AGS, Everard M, Tromp D. Élargir la pratique pharmaceutique : recentrer les soins sur les patients. Genève (Suisse) et La Haye (Pays-Bas) : Organisation mondiale de la Santé et Fédération internationale pharmaceutique; 2006. Publié au : www.fip.org/files/fip/publications/DevelopingPharmacyPractice/Developing PharmacyPracticeFR.pdf. Consulté le 3 mars 2014.

18. Principes directeurs applicables aux dons de médicaments. Ottawa $(\mathrm{ON})$ : Gouvernement du Canada; 2012. Publié au : www.acdi-cida.gc.ca/ acdi-cida/acdi-cida.nsf/fra/JUD-627121813-M5F. Consulté le 3 mars 2014.

19. Principes directeurs applicables aux dons de médicaments. Genève (Suisse) : Organisation mondiale de la Santé; 1999. Disponible à : http://libdoc.who.int/ hq/1999/WHO_EDM_PAR_99.4_fre.pdf. Consulté le 3 mars 2014.

20. Rowlands J, pour le Permanent Forum on International Pharmaceutical Crime. A guide to investigating counterfeit medical product and pharmaceutical crime. Genève (Suisse) : Organisation mondiale de la Santé et International Medical Products Anti-Counterfeiting Taskforce. Publié au : www.who.int/impact/events/Aguidetocounterfeitpharmaceutical crimeinvestigation.pdf. Consulté le 3 mars 2014.

21. Certificat en coopération internationale. Montréal (QC) : Université de Montréal, Faculté d'éducation permanente; 2014. Publié au : www.fep. umontreal.ca/cooperation/. Consulté le 3 mars 2014.

Jean-François Bussières, BPharm, MSc, MBA, FCSHP, est chef, Unité de recherche en pratique pharmaceutique et Département de pharmacie, Centre hospitalier universitaire Sainte-Justine, et professeur, Faculté de pharmacie, Université de Montréal, Montréal, Québec. II est aussi pharmacien pour le Programme de santé intégré Haïti, CHU SainteJustine.

Viriginie Bussières est diplômée d'études collégiales en sciences, Collège Jean-de Brébeuf, Montréal, Québec et stagiaire en coopération internationale.

Jean Lafontant Maurice, MD, est médecin et chirurgien en Haïti. II est aussi assistant terrain pour le Centre hospitalier universitaire Sainte-Justine, Montréal, Québec.

Martine Legault, B. Sc. Inf., M. Sc., est coordonnatrice de projet et expert clinique en soins infirmiers, Programme de santé intégré Haïti, Centre hospitalier universitaire Sainte-Justine, Montréal, Québec.

Intérêts concurrents : Aucun déclaré.

Adresse de correspondance :

Jean-François Bussières

Département de pharmacie

Centre hospitalier universitaire Sainte-Justine

3175, chemin de la Côte Sainte-Catherine

Montréal QC H3T 1 C5

Courriel : jf.bussieres@ssss.gouv.qc.ca

Remerciements : Nous tenons à remercier toute l'équipe de I'Hôpital Saint-Michel, et particulièrement le $D^{r}$ Newton Jeudy, directeur général, l'équipe de la pharmacie incluant Vital Phenel, pharmacien, Osna Victor, coordonnatrice du circuit des médicaments et fournitures de soins et les régisseuses de la pharmacie, et tous les partenaires du CHU Sainte-Justine incluant $\mathrm{M}^{\mathrm{me}}$ Isabelle Demers et $\mathrm{D}^{\mathrm{r}}$ Dickens Saint-Vil ainsi que la CroixRouge Canadienne et Croix-Rouge Haïtienne.

Financement : Jean-François Bussières, Jean Lafontant Maurice et Martine Legault sont des professionnels de la santé rémunérés dans le cadre du Programme de santé intégré Haïti; Viriginie Bussières est une stagiaire bénévole dans le cadre du programme de santé intégré Haïti. 